\title{
Isolation of potential liver $x$ receptor alpha agonist and antioxidant compounds from Hypericum microcalycinum Boiss. \& Heldr.
}

\author{
Seçil Sarıkaya Aydın' (D), Vahap Murat Kutluay ${ }^{1}$ (D), Toshiaki Makino ${ }^{2}$ (D), Makoto Inoue ${ }^{3}$ (D), \\ Ümmühan Șebnem Harput ${ }^{4}$ (D) İclal Saraçoğlu ${ }^{1}$ (D) \\ ${ }^{1}$ Hacettepe University, Faculty of Pharmacy, Department of Pharmacognosy, Ankara, Turkey \\ ${ }^{2}$ Nagoya City University, Graduate School of Pharmaceutical Sciences, Nagoya, Japan \\ ${ }^{3}$ Aichi Gakuin University, School of Pharmacy, Laboratory of Medicinal Resources, Nagoya, Japan \\ ${ }^{4}$ Freelance Scientist, Previous address: Hacettepe University, Faculty of Pharmacy, Department of Pharmacognosy, \\ Ankara, Turkey
}

ORCID IDs of the authors: S.S.A. 0000-0003-4692-9117; V.M.K. 0000-0003-4135-3497; T.M. 0000-0002-2524-8745;

M.I. 0000-0003-0116-320X; Ü.Ș.H. 0000-0002-2641-3263; I.S. 0000-0003-0555-6262

Cite this article as: Sarikaya Aydin, S., Kutluay V. M., Makino, T., Inoue, M., Harput, U. S., \& Saracoglu, I. (2021). Isolation of potential liver $\mathrm{x}$ receptor alpha agonist and antioxidant compounds from Hypericum microcalycinum Boiss. \& Heldr. İstanbul Journal of Pharmacy, 51(1), 98-104.

\begin{abstract}
Background and Aims: Hypericum microcalycinum Boiss. \& Heldr. is used for inflammatory diseases in Anatolia. The involvement of liver X receptors (LXRs) and free radicals in inflammatory diseases, activation of LXRs, and high radical contents in cancerous tissue and organs prompted us to determine the radical scavenging and LXRa agonist activity of aqueous fraction of methanol extract and fractions of $H$. microcalycinum along with the isolation studies from active fractions.

Methods: Isolation studies were carried out on chromatographic techniques. DPPH, NO, and SO radical scavenging activity methods were used for the determination of antioxidant activity, and a LXRE reporter gene assay was used for the determination of LXRa agonist activity.

Results: While the extract showed weak LXRa agonist activity, phenolic compounds- rich fractions showed moderate activity. DPPH radical scavenging capacities of the extract and some fractions seemed to be very high as well as some isolated compounds. Bioactivity- guided studies resulted in the isolation of catechin (1), epicatechin (2), apigenin-8-C-(2-0-acetyl)glucopyranoside (3), quercetin-3-0- $\beta$-glucopyranoside (4), quercetin-3-0- $\beta$-arabinopyranoside (5), kaempferol-3-0- $\beta$ arabinopyranoside (6), luteolin-8-C- $\beta$-glucopyranoside (orientin) (7).

Conclusion: According to our results, compounds 1, 2, 4, and 5 may be responsible for the anti-inflammatory effects of $H$. microcalycinum as a function of LXRa agonist and free radical scavenging effects.
\end{abstract}

Keywords: Hypericum microcalycinum, phenolic compounds, free radical scavenging, LXRa agonist activity

\section{INTRODUCTION}

Natural products or therapeutic agents derived from natural sources have an important role in human health. In a continuation of our studies to find new bioactive compounds from herbal sources, we have focused on the Hypericum species, which are well known for their antidepressant, anti-inflammatory, antiproliferative and antimicrobial activities (Boga et al., 2016; Fobofou et al., 2015). In Anatolia, Hypericum species are used as an antispasmodic, sedative, and antihelmintic internally; antiseptic and for wound healing externally (Baytop, 1984). The constituents of the genus have been previously investigated, and several types 
of compounds were determined mainly naphthodianthrones, flavonoids, acylphloroglucinol derivatives, tannins, xanthones, and essential oils (Eroglu, Aksu, \& Mat, 2008; Zorzetto et al., 2015). As part of our continuing research for bioactive metabolites from herbal medicines, we carried out chemical and biological investigations on the aerial parts of $\mathrm{H}$. microcalycinum Boiss. \& Heldr since detailed biological activity studies on this plant are lacking in the literature. H. microcalycinum has different synonyms which are H. elongatum C. A. Mey. var. microcalycinum (Boiss.\& Heldr.) A. Ramos (Nunez, 1985) and H. hyssopifolium Chaix. subsp. elongatum (Ledeb.) Woron var. microcalycinum (Boiss.\& Heldr.) (Robson, 1980).

The role of free radicals and reactive oxygen in the species is becoming increasingly important in the pathogenesis of diabetes, arteriosclerosis, cardiovascular diseases, cancer, and several neurodegenerative disorders (Aktas, Genc, Gozcelioglu, Konuklugil, \& Harput, 2013). Nuclear receptors are one of the major targets for the development of new potential agents in diseases like inflammation, rheumatoid arthritis, obesity, diabetes, and cancer (Vedin, Gustafsson, \& Steffensen, 2013). Since nuclear receptor signaling has an important function during the burying of dead cells and suppression of inflammation, nuclear receptors such as glucocorticoid receptors, PPAR (peroxisome proliferator activated receptors), and liver $X$ receptors ( $L X R)$, are important therapeutic targets in inflammatory diseases (Szondy, Garabuczi, Joos, Tsay, \& Sarang, 2014).

Liver $X$ receptors were first identified in the mid-1990s (Fessler, 2018). The LXRs, LXRa (NR1H3), and LXRß (NR1H2) form a heterodimer with the retinoid $x$ receptor $(R X R)$, and its activity can be regulated by ligands for either LXR or RXR (Willy, 1995; Wang, Nakashima, Hirai, \& Inoue, 2019). While LXRa and LXRß have similar DNA and ligand binding domains in humans, their distribution differs in tissues. LXRa is found predominantly in liver, intestine, kidney, spleen, macrophages, and adipose tissues, whereas LXRß is found more ubiquitously (Viennois et al., 2012). Previous studies have shown that both isoforms of LXR play a role in the inhibition of some inflammatory genes such as iNOS, COX2, IL6, the chemokines monocyte chemoattractant protein-1 (MCP-1) and MCP-3, and matrix metalloproteinase-9 (MMP9) (Joseph, Castrillo, Laffitte, Mangelsdorf, \&Tontonoz, 2003).

It has been shown that LXRa has also a role in preventing oxidative stress and can affect oxidative stress response by regulating the expression of antioxidant genes (Gong et al., 2009). Nuclear receptors are one of the major targets for the development of new potential agents in diseases like inflammation, rheumatoid arthritis, obesity, diabetes, and cancer (Vedin et al., 2013).

Natural products and nature-derived compounds attract more attention day by day in the treatment of human and animals diseases. The members of the Hypericum genus have been used in the treatment of various diseases worldwide and have been traded in the global marketplace. In this study, we focus on H. microcalycinum, which has not been studied in detail, to determine the potential antioxidant and anti-inflammatory activities. We aimed to identify the anti-inflammatory potential of the extract and to determine the bioactive compounds. For this purpose, the LXR response element (LXRE) reporter gene assay (to determine LXRa agonist activity) and DPPH, NO, and SO radical scavenging activities (to determine antioxidant activity) for the aqueous fraction of methanol extract and different polyamide column fractions of $H$. microcalycinum were tested. As Hypericum species have been shown to be involved in inflammatory mechanisms, we hypothesized that it could be a function of the plant as a nuclear receptor activator and/ or the extract's antioxidant activity.

\section{MATERIALS AND METHODS}

\section{General}

Fractionation and isolation studies were carried out on chromatographic techniques such as silica gel (Kieselgel 60, 60-230 mesh, Merck, Darmstadt, Germany), polyamide (Sigma Aldrich, St. Louis, MI, USA). In medium pressure liquid chromatography (MPLC) LiChroprep C18 (40-63 $\mu \mathrm{m}$, Merck) was used as an adsorbent. The system was equipped with a Büchi column (3,5 $\mathrm{x}$ $45 \mathrm{~cm}$ ). 5-15 bar pressure and $5 \mathrm{ml} / \mathrm{min}$ flow rates were used. Silica gel 60 F254 plates (Merck) were used during isolation studies and $\mathrm{CHCl}_{3}-\mathrm{MeOH}-\mathrm{H}_{2} \mathrm{O}$ (61:32:7, 70:30:3, 80:20:2) was used as the solvent system.

NMR spectra were recorded on an Agilent Varian VNS500 spectrometer (Agilent, Santa Clara, CA, USA). Positive-mode ESITOFMS was obtained on a JEOL JMS-T100LP AccuTOF LC-plus 4G spectrometer (JEOL, Tokyo, Japan) using a sample dissolved in $\mathrm{MeOH}$.

3- (4,5-dimethylthiazol-2-yl)-2,5-diphenyltetrazolium bromide (MTT), DPPH, nitro blue tetrazolium (NBT), sodium nitroprusside, Folin-Ciocalteu reagent, gallic acid, ascorbic acid (AA) were obtained from Sigma-Aldrich. 3-t-butyl-4-hydroxyanizole (BHA) was purchased from Nacalai Tesque (Kyoto, Japan). Sulfanilamide and naphthylethylenediamine dihydrochloride were obtained from Merck.

HEK293 (Human embryonic kidney) cell line was obtained from Riken Bioresource Center Cell Bank (Japan). Minimum Essential Medium Earle's salts (MEM's Earle), trypsin were obtained from Sigma-Aldrich. Fetal bovine serum (FBS) was purchased from Biowest (Nuaillé, France). Penicillin, streptomycin, and MEM non-essential amino acid solution were obtained from Fujifilm Wako Pure Chemical Industries (Osaka, Japan). T0901317 was purchased from Cayman Chemical (Ann Arbor, MI, USA).

\section{Plant material}

The aerial parts of H. microcalycinum were collected from AğrıDoğubeyazıt in July 2011 and identified by Prof. Dr. Hayri Duman (Gazi University, Faculty of Science, Department of Botany, Ankara, Turkey). A voucher specimen has been deposited in the Herbarium of the Faculty of Pharmacy, Hacettepe University, Ankara, Turkey (HUEF 11006).

\section{Extraction and isolation}

The air-dried aerial parts of the plant $(90.8127 \mathrm{~g})$ were extracted with $\mathrm{MeOH}$ at $40^{\circ} \mathrm{C}$ for $12 \mathrm{~h}(4 \times 400 \mathrm{~mL})$. A rotary equipped with a water bath and a $2 \mathrm{~L}$ flask was used for the extraction. The $\mathrm{MeOH}$ solutions were evaporated under vacuum to yield $\mathrm{MeOH}$ extract and it was dissolved in water and partitioned with petroleum ether to remove chlorophylls and other lipophilic compounds. 
The aqueous fraction was lyophilized to yield $12.85 \mathrm{~g}$ dry weight. The aqueous fraction was applied to polyamide column chromatography to get four sub-fractions using increasing concentrations of methanol (0-25-50-75-100\%). The aqueous fraction of methanol extract and polyamide column fractions were tested for radical-scavenging and LXRa agonist activities. Further isolation studies were continued on the active and phenolic compound rich fraction (Fraction D; 795 mg). Fr. D was applied to MPLC using $0-100 \%$ methanol as a solvent system. Five sub-fractions were obtained, and the fraction of $27 \%$ methanol was applied to silica gel column chromatography using different concentrations of $\mathrm{CHCl}_{3}: \mathrm{CH}_{3} \mathrm{OH}$ as a mobile phase to give compounds $\mathbf{1}$ and $\mathbf{2}$ as a mixture (22.6 mg). The fraction of $45 \%$ methanol was applied to silica gel column chromatography using different concentrations of $\mathrm{CHCl}_{3}: \mathrm{CH}_{3} \mathrm{OH}$ as a mobile phase to give compound $\mathbf{3}$ in pure form (18.0 mg) and two of MPLC fractions were applied to preparative TLC to get compound $\mathbf{4}(20.3 \mathrm{mg})$ and compound $\mathbf{5}$ (70.8 $\mathrm{mg}$ ) in pure form. MPLC fraction of $45 \%$ methanol was applied to preparative TLC to get compound $\mathbf{6}(3.4 \mathrm{mg})$ in pure form. MPLC fraction of $40 \%$ methanol was applied to preparative TLC to get compound 7 (5.1 mg) in pure form.

\section{Antioxidant activity}

\section{DPPH radical scavenging activity}

The DPPH radical scavenging effect was assessed by the discoloration of methanol solution of DPPH spectroscopically; AA and BHA were used as positive controls (Hatano et al., 1989; Jensen, Gotfredsen, Harput, \& Saracoglu, 2010). DPPH (1 mM) solution was added to the $\mathrm{MeOH}$ solution of the extract, fractions, or compounds at various concentrations. The reaction mixture was shaken vigorously, and the absorbance of the remaining DPPH was measured at $517 \mathrm{~nm}$ after $30 \mathrm{~min}$.. All the analyses were done in triplicate. Radical scavenging activity was expressed as the inhibition percentage.

\section{SO radical scavenging activity by alkaline DMSO method}

The method of Kunchandy and Rao was used to detect SO radical-scavenging activity of the samples, with slight modification. In brief, a SO radical was generated in a nonenzymatic system. The reaction mixture containing nitro blue tetrazolium (1 mg/ $\mathrm{mL}$ solution in DMSO) and the sample or standard compounds was dissolved in DMSO. A total of $100 \mu \mathrm{L}$ of alkaline DMSO $(1 \mathrm{~mL}$ DMSO containing $5 \mathrm{mM} \mathrm{NaOH}$ in $0.1 \mathrm{~mL}$ water) was added to yield a final volume of $140 \mu \mathrm{L}$. The absorbance was measured at 560 nm by using a microplate reader (Kunchandy \& Rao, 1990; Srinivasan, Chandrasekar, Nanjan, \& Suresh, 2007).

\section{NO radical scavenging activity}

To determine NO radical scavenging activity of the samples, a serial diluted sample was added to a 96-well flat-bottomed plate. $10 \mathrm{mM}$ sodium nitroprusside, dissolved in phosphate-buffered saline, was added to each well and the plate was incubated under light at room temperature for 150 minutes. Finally, an equal volume of the Griess reagent ( $1 \%$ sulfanilamide, $0.1 \%$ naphthylethylenediamine dihydrochloride, $\left.2.5 \% \mathrm{H}_{3} \mathrm{PO}_{4}\right)$ was added to each well to measure the nitrite content. After chromophore was formed at room temperature in 10 minutes, absorbance at $577 \mathrm{~nm}$ was measured on a microplate reader (Tsai, Tsai, Yu, \& Ho, 2007).

\section{LXRa agonist activity}

The LXRE reporter gene assay was used to determine LXRa agonist activity. HEK293 cells were cultured in MEM (Minimum Essential Medium) supplemented with 10\% FBS, 1\% nonessential amino acids, $50 \mathrm{U} / \mathrm{ml}$ penicillin, and $50 \mu \mathrm{g} / \mathrm{ml}$ streptomycin at $37{ }^{\circ} \mathrm{C}$ in a humidified atmosphere of $5 \% \mathrm{CO}_{2}$ in the air.

The calcium phosphate co-precipitation method was used for transfection. For LXR luciferase reporter assay, pBApo-CMWhLXR-a (30 ng), pGL4.1-DR4-Luc (120 ng), pCMX- $\beta$-gal expression vector (30 ng), and carrier DNA pUC18 were used to yield a total of $600 \mathrm{ng}$ of DNA per well. After 6 hours, the cells were treated with test samples for 36 hours of incubation. The luciferase and $\beta$-galactosidase activities were analyzed from cell lysates using a luminescence reader and a spectrophotometer, respectively. The results were given in fold induction values relative to vehicle-treated cells after normalization of luciferase activity by $\beta$-galactosidase (Kotani, Tanabe, Mizukami, Makishima, \& Inoue, 2010; Nakashima, Murakami, Tanabe, \& Inoue, 2014).

\section{RESULTS AND DISCUSSION}

The methanol extract of H. microcalycinum aerial part was suspended in water and partitioned with petroleum ether. The aqueous fraction was used for the phytochemical and biological analysis. The aqueous fraction was subjected to polyamide column chromatography to afford four main fractions.

To evaluate LXRa agonist activity, the aqueous fraction of H. microcalycinum extract and its main polyamide column fractions were tested at a concentration of $100 \mu \mathrm{g} / \mathrm{mL}$. In this study, an LXRa agonist, T09011317, was used as a positive control at the concentrations of 1, 10 and $100 \mathrm{nM}$. The results were given as fold induction values normalized by $\beta$-galactosidase. While the extract showed weak LXRa agonist activity, Fr. D, the phenolic compound-rich fraction, showed moderate activity with a fold value of 1.37 (Figure 1, Table 1).

\section{Table 1. LXRa agonist activities of the Hypericum microcalycinum extract and fractions* Effects of Hypericum microcalycinum (aerial parts) and fractions on LXRE reporter gene transcription at 100 $\mu \mathrm{g} / \mathrm{ml}$ concentration were given as fold values.}

\begin{tabular}{lc} 
Samples & Fold Value \\
\hline Aqueous extract & $0.96 \pm 0.02$ \\
Fr. A & $1.15 \pm 0.06$ \\
Fr. B & $1.6 \pm 0.06$ \\
Fr. C & $2.22 \pm 0.07$ \\
Fr. D & $1.37 \pm 0.08$ \\
T0901317 1 nM & $1.22 \pm 0.11$ \\
T0901317 10 nM & $2.91 \pm 0.13$ \\
T0901317 100 nM & $5.49 \pm 0.34$ \\
Control & $1 \pm 0.03$
\end{tabular}

*Three independent test results were considered, averages and standard error means were given in the table. 


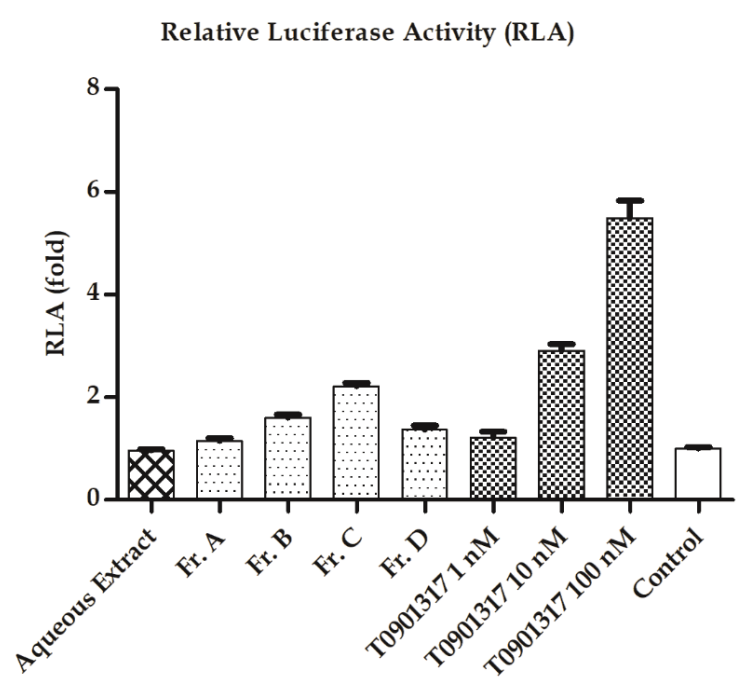

Figure 1. Effects of Hypericum microcalycinum (aerial parts) on LXRE reporter gene transcription at $100 \mu \mathrm{g} / \mathrm{mL}$ concentration. Three independent test results were considered, averages and standard error means were given in the figure.

The aqueous H. microcalycinum extract and fractions of polyamide column were tested against 2,2-diphenyl-1-picrylhydrazyl (DPPH), NO, and SO radicals for antioxidant activity. Results were evaluated with the known antioxidants ascorbic acid (AA), butyl-4-hydroxyanizole (BHA), and quercetin. Tested fractions showed radical scavenging activity depending on their phenolic contents. Fr. A is the non-phenolic fraction of the polyamide column that showed the lowest radical scavenging activity. Fr. $D$, the phenolic- rich fraction, was found to possess high radical scavenging activity against the tested radicals. Its radical scavenging activity was comparable to that of known antioxidants $\mathrm{AA}, \mathrm{BHA}$, and quercetin. Radical scavenging activity (inhibition \%) of Fr. D at a concentration of $200 \mu \mathrm{g} / \mathrm{mL}$ against DPPH, NO and SO radicals were found as 90.5, 59.9, and 65.5 respectively, w hereas radical scavenging activity (inhibition \%) of Fr. A at a concentration of $200 \mu \mathrm{g} / \mathrm{mL}$ against DPPH, NO and SO radicals were found as $0.8,24.4$, and 42.1 respectively.

Repeated chromatographies of the flavonoid fraction (Fr. D), which wa s eluted with $75-100 \%$ methanol, resulted in the isolation of seven compounds. The spectroscopic data (1D and 2D-NMR, and ESITOFMS) of the isolated compounds were compared to the data of the compounds that were given in the references and their structures were identified as follows: Catechin (1) and epicatechin (2) (Donovan, Luthria, Stremple, \& Waterhouse, 1999), apigenin-8-C-(2-O-acetyl)-glucopyranoside (3) (Zhang \& Xu, 2003), quercetin-3-O- $\beta$-glucopyranoside (4) (Islam et al., 2012), quercetin-3-O- $\alpha$-arabinopyranoside (5) (Kadota et al., 1990), kaempferol-3-O- $\beta$-arabinopyranoside (6) (Nicollier \& Thompson, 1983), luteolin-8-C- $\beta$-glucopyranoside (orientin) (7) (Almahy \& Fouda, 2013) (Figure 2). All data is provided in the Supplementary document. Catechin and epicatechin were obtained as a mixture (ratio 1:3). Apigenin-8-C-(2-Oacetyl)- $\beta$-glucopyranoside was isolated for the first time from a Hypericum species in this study.
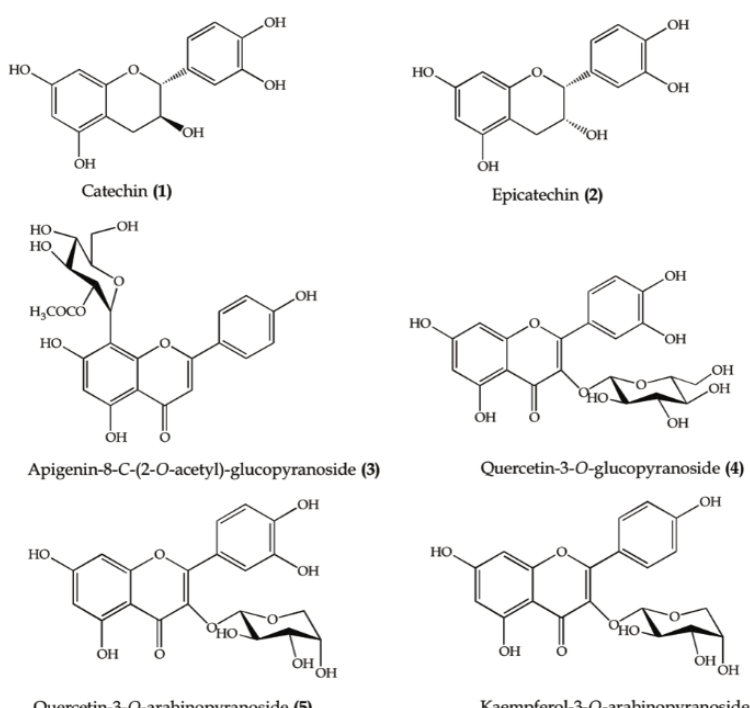

Kaempferol-3-O-arabinopyranoside (6)

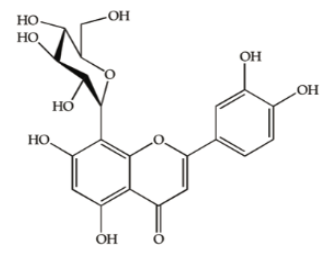

Luteolin-8-C-glucopyranoside (Orientin) (7)

Figure 2. Isolated compounds from Hypericum microcalycinum Boiss.\&Heldr.

Catechin (1): ${ }^{1} \mathrm{H}$ NMR $\left(500 \mathrm{MHz}, \mathrm{CD}_{3} \mathrm{OD}\right): 6.87(1 \mathrm{H}, \mathrm{d}, \mathrm{j}=1.9$, $\left.H-2^{\prime}\right), 6.80\left(1 \mathrm{H}, \mathrm{dd}, j=8.0 \mathrm{~Hz}, \mathrm{H}-5^{\prime}\right), 6.76\left(1 \mathrm{H}, \mathrm{dd}, \mathrm{j}=8.2 / 1.3, \mathrm{H}-6^{\prime}\right)$, $5.96(1 \mathrm{H}, \mathrm{d}, \mathrm{j}=2.31 \mathrm{~Hz}, \mathrm{H}-8), 5.89(1 \mathrm{H}, \mathrm{d}, j=2.31 \mathrm{~Hz}, \mathrm{H}-6), 4.59$ $(1 \mathrm{H}, \mathrm{d}, \mathrm{j}=7.5 \mathrm{~Hz}, \mathrm{H}-2), 4.00(1 \mathrm{H}, \mathrm{ddd}, \mathrm{H}-3), 2.54-2.90(2 \mathrm{H}, \mathrm{dd}$, $\mathrm{H}-4),{ }^{13} \mathrm{C}$ NMR (125 MHz, CD OD): 157.86 (C-7), 157.59 (C-9), $156.93(C-5), 146.26\left(C-3^{\prime}\right), 146.24\left(C-4^{\prime}\right), 132.30\left(C-1^{\prime}\right), 120.04$ $\left(C-6^{\prime}\right), 116.08\left(C-5^{\prime}\right), 115.27\left(C-2^{\prime}\right), 100.82(C-10), 96.29(C-8)$, 95.50 (C-6), 82.87 (C-2), $68.83(\mathrm{C}-3), 28.54$ (C-4)

Epicatechin (2): ${ }^{1} \mathrm{H}$ NMR $\left(500 \mathrm{MHz}, \mathrm{CD}_{3} \mathrm{OD}\right): 7.01(1 \mathrm{H}, \mathrm{d}, \mathrm{j}=$ $\left.1.9, H-2^{\prime}\right), 6.84\left(1 \mathrm{H}, \mathrm{dd}, j=8.4 / 1.8, \mathrm{H}-6^{\prime}\right), 6.79(1 \mathrm{H}, \mathrm{dd}, j=8.1 \mathrm{~Hz}$, $\left.\mathrm{H}-5^{\prime}\right), 5.98(1 \mathrm{H}, \mathrm{d}, j=2.37 \mathrm{~Hz}, \mathrm{H}-8), 5.95(1 \mathrm{H}, \mathrm{d}, j=2.29 \mathrm{~Hz}, \mathrm{H}-6)$, $4.85(1 \mathrm{H}, \mathrm{s}, \mathrm{H}-2), 4.21(1 \mathrm{H}, \mathrm{m}, \mathrm{H}-3), 2.77-2.88(2 \mathrm{H}, \mathrm{dd}, \mathrm{H}-4),{ }^{13} \mathrm{C}$ $\operatorname{NMR}\left(125 \mathrm{MHz}, \mathrm{CD}_{3} \mathrm{OD}\right): 158.02(\mathrm{C}-7), 157.69$ (C-9), 157.38 (C-5), $145.96\left(C-3^{\prime}\right), 145.80\left(C-4^{\prime}\right), 132.24\left(C-1^{\prime}\right), 119.40\left(C-6^{\prime}\right), 115.89$ $\left(\mathrm{C}-5^{\prime}\right), 115.33\left(\mathrm{C}-2^{\prime}\right), 100.07(\mathrm{C}-10), 96.39(\mathrm{C}-6), 95.87$ (C-8), 79.90 $(C-2), 67.50(C-3), 29.27$ (C-4)

Apigenin-8-C-(2-O-acetyl) glucopyranoside (3): ' ${ }^{1} \mathrm{H}$ NMR (500 $\left.\mathrm{MHz}, \mathrm{CD}_{3} \mathrm{OD}\right): 8.07\left(1 \mathrm{H}, \mathrm{d}, \mathrm{j}=8.8 \mathrm{~Hz}, \mathrm{H}-2^{\prime} / 6^{\prime}\right), 6.99(1 \mathrm{H}, \mathrm{d}, \mathrm{j}=$ $\left.8.8 \mathrm{~Hz}, \mathrm{H}-3^{\prime} / 5^{\prime}\right), 6.64(1 \mathrm{H}, \mathrm{s}, \mathrm{H}-3), 6.25(1 \mathrm{H}, \mathrm{s}, \mathrm{H}-6), 5.57(1 \mathrm{H}$, $\left.\mathrm{dd} j=4.5 / 10.2 \mathrm{~Hz}, \mathrm{H}-2^{\prime \prime}\right), 5.11\left(1 \mathrm{H}, \mathrm{d}, j=10.2 \mathrm{~Hz}, \mathrm{H}-1^{\prime \prime}\right), 4.02$ $\left(1 \mathrm{H}, \mathrm{dd}, j=2.1 / 12.1, \mathrm{H}-6^{\prime \prime} \mathrm{a}\right), 3.86\left(1 \mathrm{H}, \mathrm{dd}, \mathrm{j}=5.7 / 12.1, \mathrm{H}-6^{\prime \prime} \mathrm{b}\right)$, $3.74\left(1 \mathrm{H}, \mathrm{d}^{*}, \mathrm{H}-3^{\prime \prime}\right), 3.74\left(1 \mathrm{H}, \mathrm{d}, \mathrm{H}-4^{\prime \prime}\right), 3.54\left(1 \mathrm{H}, \mathrm{m}, \mathrm{H}-5^{\prime \prime}\right), 1.70$ ( $3 \mathrm{H}, \mathrm{s}, \mathrm{CH} 3) ;{ }^{13} \mathrm{C}$ NMR $\left(125 \mathrm{MHz}, \mathrm{CD}_{3} \mathrm{OD}\right): 184.15$ (C-4), 171.91 $(C=0), 166.71(C-2), 163.90(C-7), 162.99(C-5), 162.76\left(C-4^{\prime}\right)$, $158.58(C-9), 130.13\left(C-2^{\prime} / 6^{\prime}\right), 123.66\left(C-1^{\prime}\right), 116.97\left(C-3^{\prime} / 5^{\prime}\right)$, 105.75 (C-10), 103.66 (C-3), 103.60 (C-8), 98.97 (C-6), 83.14 (C$\left.5^{\prime \prime}\right), 77.74\left(C-3^{\prime \prime}\right), 74.08\left(C-2^{\prime \prime}\right), 73.00\left(C-1^{\prime \prime}\right), 72.24\left(C-4^{\prime \prime}\right), 62.93$ $\left(\mathrm{C}-6^{\prime \prime}\right), 20.52(\mathrm{CH} 3)$. 
Quercetin-3-O-glucopyranoside (4): ${ }^{1} \mathrm{H}$ NMR $(500 \mathrm{MHz}$, $\left.\mathrm{CD}_{3} \mathrm{OD}\right): 7.75\left(1 \mathrm{H}, \mathrm{d}, \mathrm{j}=2.1 \mathrm{~Hz}, \mathrm{H}-2^{\prime}\right), 7.61(1 \mathrm{H}, \mathrm{dd}, \mathrm{j}=2.1 / 8.4 \mathrm{~Hz}$, $\left.H-6^{\prime}\right), 6.88\left(1 \mathrm{H}, \mathrm{d}, \mathrm{j}=8.4 \mathrm{~Hz}, \mathrm{H}-5^{\prime}\right), 6.26(1 \mathrm{H}, \mathrm{d}, \mathrm{j}=1.8 \mathrm{~Hz}, \mathrm{H}-8)$, $6.11(1 \mathrm{H}, d, j=1.8 \mathrm{~Hz}, \mathrm{H}-6), 5.15\left(1 \mathrm{H}, \mathrm{d}, j=7.8 \mathrm{~Hz}, \mathrm{H}-1^{\prime \prime}\right), 3.74(1 \mathrm{H}$, $\left.\mathrm{dd}, j=2.3 / 11.9 \mathrm{~Hz}, \mathrm{H}-6^{\prime \prime} \mathrm{a}\right), 3.62\left(1 \mathrm{H}, \mathrm{dd}, \mathrm{j}=5.2 / 11.9 \mathrm{~Hz}, \mathrm{H}-6^{\prime \prime} \mathrm{b}\right)$, $3.52\left(1 \mathrm{H}, d d, j=7.8 / 9.0 \mathrm{~Hz}, \mathrm{H}-2^{\prime \prime}\right), 3.45\left(1 \mathrm{H}, \mathrm{m}, \mathrm{H}-3^{\prime \prime}\right), 3.39(1 \mathrm{H}$, $\left.\mathrm{m}, \mathrm{H}-4^{\prime \prime}\right)$, $3.25\left(1 \mathrm{H}, \mathrm{m}, \mathrm{H}-5^{\prime \prime}\right),{ }^{13} \mathrm{C}$ NMR (125 MHz, CD $\left.\mathrm{OD}\right): 178.60$ (C-4), 170.30 (C-7), 162.59 (C-5), 158.99 (C- 2), 158.13 (C-9), $150.45\left(C-4^{\prime}\right), 146.06\left(C-3^{\prime}\right), 135.39(C-3), 123.07\left(C-6^{\prime}\right), 122.96$ $\left(C-1^{\prime}\right), 117.30\left(C-2^{\prime}\right), 116.05\left(C-5^{\prime}\right), 105.09\left(C-1^{\prime \prime}\right), 103.65(C-10)$, 102.20 (C-6), $96.33(\mathrm{C}-8), 78.33\left(\mathrm{C}-5^{\prime \prime}\right), 78.20\left(\mathrm{C}-3^{\prime \prime}\right), 75.02\left(\mathrm{C}-2^{\prime \prime}\right)$, $71.14\left(C-4^{\prime \prime}\right), 62.53\left(C-6^{\prime \prime}\right)$.

Quercetin-3-O-arabinopyranoside (5): ${ }^{1} \mathrm{H}$ NMR $(500 \mathrm{MHz}$, $\left.\mathrm{CD}_{3} \mathrm{OD}\right): 7.78\left(1 \mathrm{H}, \mathrm{d}, \mathrm{j}=2.1 \mathrm{~Hz}, \mathrm{H}-2^{\prime}\right), 7.61(1 \mathrm{H}, \mathrm{dd}, \mathrm{j}=2.1 / 8.5 \mathrm{~Hz}$, $\left.\mathrm{H}-6^{\prime}\right), 6.91\left(1 \mathrm{H}, \mathrm{d}, \mathrm{j}=8.5 \mathrm{~Hz}, \mathrm{H}-5^{\prime}\right), 6.44(1 \mathrm{H}, \mathrm{d}, \mathrm{j}=2.1 \mathrm{~Hz}, \mathrm{H}-8)$, $6.24(1 \mathrm{H}, \mathrm{d}, \mathrm{j}=2.1 \mathrm{~Hz}, \mathrm{H}-6), 5.20\left(1 \mathrm{H}, \mathrm{d}, \mathrm{j}=6.5 \mathrm{~Hz}, \mathrm{H}-1^{\prime \prime}\right), 3.94(1 \mathrm{H}$, $\left.d d, j=6.5 / 8.4 \mathrm{~Hz}, \mathrm{H}-2^{\prime \prime}\right), 3.89\left(1 \mathrm{H}, \mathrm{m}, \mathrm{H}-5^{\prime \prime} \mathrm{a}\right), 3.88\left(1 \mathrm{H}, \mathrm{brs}, \mathrm{H}-4^{\prime \prime}\right)$, $3.68\left(1 \mathrm{H}, \mathrm{dd}, \mathrm{j}=3.2 / 8.4, \mathrm{H}-3^{\prime \prime}\right), 3.48\left(1 \mathrm{H}, \mathrm{d}, \mathrm{j}=10.4, \mathrm{H}-5^{\prime \prime} \mathrm{b}\right) ;{ }^{13} \mathrm{C}$ NMR (125 MHz, CD OD): 179.49 (C-4), 164.07 (C-7), 163.07 (C5), $158.71(C-9), 158.44(C-2), 149.96\left(C-4^{\prime}\right), 145.99\left(C-3^{\prime}\right), 135.65$ (C-3), $123.03\left(\mathrm{C}-6^{\prime}\right), 122.89\left(\mathrm{C}-1^{\prime}\right), 117.45\left(\mathrm{C}-2^{\prime}\right), 116.17\left(\mathrm{C}-5^{\prime}\right)$, 105.65 (C-10), $104.62\left(C-1^{\prime \prime}\right), 99.87(C-6), 94.69(C-8), 74.12$ (C$\left.3^{\prime \prime}\right), 72.87\left(C-2^{\prime \prime}\right), 69.10\left(C-4^{\prime \prime}\right), 66.94\left(C-5^{\prime \prime}\right)$.

Kaempferol-3-O-arabinopyranoside (6): 'H NMR (500 MHz, $\left.\mathrm{CD}_{3} \mathrm{OD}\right): 6.15(1 \mathrm{H}, \mathrm{d}, \mathrm{j}=2.0 \mathrm{~Hz}, \mathrm{H}-6), 6.02(1 \mathrm{H}, \mathrm{d}, \mathrm{j}=2.0 \mathrm{~Hz}$, $\mathrm{H}-8), 8.05\left(1 \mathrm{H}, \mathrm{d}, \mathrm{j}=8.8 \mathrm{~Hz}, \mathrm{H}-2^{\prime} / \mathrm{H}-6^{\prime}\right), 6.85(1 \mathrm{H}, \mathrm{d}, \mathrm{j}=8.7 \mathrm{~Hz}$, $\left.\mathrm{H}-3^{\prime} / \mathrm{H}-5^{\prime}\right), 4.95\left(1 \mathrm{H}, \mathrm{d}, \mathrm{j}=6.5, \mathrm{H}-1^{\prime \prime}\right), 3.92\left(1 \mathrm{H}, \mathrm{dd}, \mathrm{j}=8.4 / 6.5, \mathrm{H}-2^{\prime \prime}\right)$, $3.65\left(1 \mathrm{H}, \mathrm{dd}, \mathrm{j}=3.4 / 8.3, \mathrm{H}-3^{\prime \prime}\right), 3.81\left(1 \mathrm{H}, \mathrm{m}, \mathrm{H}-4^{\prime \prime}\right), 3.46(1 \mathrm{H}, \mathrm{dd}$, $\left.j=12.5 / 1.0, H-5^{\prime \prime} b\right), 3.83\left(1 \mathrm{H}, \mathrm{d}, \mathrm{j}=12, \mathrm{H}-5^{\prime \prime} \mathrm{a}\right) ;{ }^{13} \mathrm{C}$ NMR $(125 \mathrm{MHz}$, $\left.\mathrm{CD}_{3} \mathrm{OD}\right): 158.5$ (C- $\left.2 \mathrm{j}\right), 135.5$ (C-3), 170.29 (C-4), 159.31 (C-5), 97.40 (C-6), 162.36 (C-7), 103.62 (C-8), 154.81 (C-9), 102.50 (C10), $122.1\left(C-1^{\prime}\right), 131.97\left(C-2^{\prime}\right), 116.89\left(C-3^{\prime}\right), 163.2\left(C-4^{\prime}\right), 116.89$ $\left(C-5^{\prime}\right), 131.97\left(C-6^{\prime}\right), 105.40\left(C-1^{\prime \prime}\right), 72.80\left(C-2^{\prime \prime}\right), 74.33\left(C-3^{\prime \prime}\right)$, $69.12\left(C-4^{\prime \prime}\right), 66.92\left(C-5^{\prime \prime}\right)$.

Luteolin-8-C-glucopyranoside (Orientin) (7): ' H NMR (500 MHz, $\left.\mathrm{CD}_{3} \mathrm{OD}\right): 7.56\left(1 \mathrm{H}, \mathrm{br} . \mathrm{S}, \mathrm{H}-2^{\prime}\right), 7.51\left(1 \mathrm{H}, \mathrm{dd}, \mathrm{j}=8.2, \mathrm{H}-6^{\prime}\right), 6.92(1 \mathrm{H}$, $\left.d, j=8.2, H-5^{\prime}\right), 6.51(1 \mathrm{H}, \mathrm{s}, \mathrm{H}-3), 6.23(1 \mathrm{H}, \mathrm{s}, \mathrm{H}-6), 5.05(1 \mathrm{H}, \mathrm{d}, \mathrm{j}=$ 9.9, $\left.\mathrm{H}-1^{\prime \prime}\right), 4.15\left(1 \mathrm{H}, \mathrm{t}, \mathrm{H}-2^{\prime \prime}\right), 3.97\left(1 \mathrm{H}, \mathrm{d}, \mathrm{j}=10.6, \mathrm{H}-6^{\prime \prime} \mathrm{a}\right), 3.86^{*}$ $\left(1 \mathrm{H}, \mathrm{H}-6^{\prime \prime} b\right), 3.69\left(1 \mathrm{H}, \mathrm{m}, \mathrm{H}-4^{\prime \prime}\right), 3.60\left(1 \mathrm{H}, \mathrm{m}, \mathrm{H}-3^{\prime \prime}\right), 3.53(1 \mathrm{H}$, m, H-5"); ${ }^{13} \mathrm{C}$ NMR (125 MHz, CD $\mathrm{OD}$ ): 183.68 (C-4), 166.30 (C7), $166.30(C-2), 162.49(C-5), 158.0(C-9), 151.72\left(C-4^{\prime}\right), 147.34$ $\left(C-3^{\prime}\right), 123.72\left(C-1^{\prime}\right), 120.73\left(C-6^{\prime}\right), 116.80\left(C-5^{\prime}\right), 114.53\left(C-2^{\prime}\right)$, 104.48 (C-3), $104.0(C-10), 103.62(C-8), 97.40(C-6), 82.83\left(C-5^{\prime \prime}\right)$, $80.48\left(C-3^{\prime \prime}\right), 75.5\left(C-1^{\prime \prime}\right), 72.90\left(C-2^{\prime \prime}\right), 72.50\left(C-4^{\prime \prime}\right), 63.10\left(C-6^{\prime \prime}\right)$.

As seen in Table 2, five of the isolated compounds were tested for their radical scavenging activity because of their adequate amount for testing. While four of them showed strong scavenging activity, compounds $\mathbf{1}$ and $\mathbf{2}$, which were a 1:3 mixture of catechin-epicatechin, exhibited the strongest free radical scavenging activity. Its activity was found to be more potent or very close to that of $\mathrm{AA}, \mathrm{BHA}$, and quercetin in the case of nitric oxide and superoxide radicals.

Catechin and epicatechin have shown high antioxidant activity due to their high number of hydroxyl groups (Prochazkova, Bousova, \&Wilhelmova, 2011). In previous studies have report-

\section{Table 2. Radical scavenging activities of the Hypericum microcalycinum extract and isolated compounds*.}

\begin{tabular}{|lccc|}
\hline & \multicolumn{2}{c}{ Radical scavenging $\mathrm{IC}_{\mathbf{5 0}}(\mu \mathrm{g} / \mathrm{ml})^{\mathrm{a}}$} \\
\hline Samples & DPPH & NO & SO \\
\hline $\begin{array}{l}\text { Aqueous } \\
\text { extract }\end{array}$ & $51.8 \pm 0.86$ & $>1000$ & $134.5 \pm 3.57$ \\
$\begin{array}{l}\text { Compounds } \mathbf{1} \\
\text { and 2 }\end{array}$ & $23.1 \pm 0.26$ & $38.9 \pm 5.71$ & $33.5 \pm 1.11$ \\
$\begin{array}{l}\text { Compound } \mathbf{3} \\
\text { Compound } \mathbf{4}\end{array}$ & $358.8 \pm 6.36$ & $85.5 \pm 1.04$ & $>800$ \\
Compound $\mathbf{5}$ & $25.5 \pm 0.18$ & $61.8 \pm 4.33$ & $72.7 \pm 4.59$ \\
AA & $9.1 \pm 0.08$ & $66.6 \pm 4.49$ & $25.2 \pm 0.81$ \\
BHA & $6.7 \pm 0.17$ & $310.7 \pm 10.34$ & $>800$ \\
Quercetin & $6.2 \pm 0.04$ & $55.3 \pm 1.89$ & $14.3 \pm 0.73$ \\
\hline
\end{tabular}

a Radical scavenging activities of the isolated compounds and the references on DPPH, NO and SO radicals (BHA: butyl-4-hydroxyanizole; $A$ A: ascorbic acid); ${ }^{\circ}$ Compounds $\mathbf{1}$ and $\mathbf{2}$ were isolated as a mixture (ratio 1:3); * Compounds $\mathbf{6}$ and $\mathbf{7}$ were not assessed because of the limited amount of sample; Three independent test results were considered, averages and standard deviations were given in table.

ed that the o-dihydroxy group in the $\mathrm{B}$ ring of flavonoids and the functional hydroxyl groups in the 3-and 5- positions also mediated antioxidant activity. Glycosylation at position 3 also decreases the radical scavenging (Prochazkova et al., 2011). The results of our study supported these data. According to the present research, it was found that compounds 1, 2, 4, and 5 show higher antioxidant activity. Comparison results of compounds 4 and 5 with quercetin support that glycosylation reduces the antioxidant effect. The results of DPPH radical scavenging assay in this study were compared to results obtained from previous literature. The $\mathrm{IC}_{50}$ values of compounds 1 and 2 (catechin-epicatechin mixture), compound 4 (quercetin-3-Oglucopyranoside), compound 5 (quercetin-3-O-arabinopyranoside), and the positive control (quercetin) were found as 23.1, $53.2,25.5$, and $6.2 \mu \mathrm{g} / \mathrm{mL}$, respectively. These data were similar to those in previous literature, in which $\mathrm{IC}_{50}$ values of catechin, epicatechin, quercetin-3-O-glucopyranoside, quercetin-3-0arabinopyranoside, and quercetin were reported as 14.3, 9.9, 22, 27.9, and $7.7 \mu \mathrm{g} / \mathrm{mL}$, respectively (Razavi, Zahri, Zarrini, Nazemiyeh, \& Mohammadi, 2009; Sarian et al., 2017; Zhang et al., 2005).

Previous studies on the structure-activity relationship studies of flavonoids suggested that hydroxyl group at position 3 could be important to activate LXRa. For example, while quercetin acts as an agonist, luteolin shows antagonist activity for LXRa (Fouache et al., 2019; Francisco et al., 2016). The hydroxyl group at the positions 5,7 and 4' are also necessary to show agonist activity (Fouache et al., 2019). The studies also revealed that not only aglycons, but also flavonoid glycosides could also activate LXRa (Hiebl, Ladurner, Latkolik, \& Dirsch, 2018). Kaempferol-3-O- $\beta$-glucopyranoside also showed potent activity for LXRa with an $\mathrm{EC}_{50}$ value of $1.8 \mu \mathrm{M}$. Quercetin-3-O-gluc- 
uronide was also shown to activate LXRa (Ohara et al., 2013). These findings suggest that compounds $\mathbf{4}, \mathbf{5}$, and $\mathbf{6}$ might be responsible for the LXRa agonist activity of the extract.

\section{CONCLUSION}

Hypericum species has widespread use in traditional and contemporary medicine for its antidepressant, anti-inflammatory, antiproliferative and antimicrobial activities. According to our results, isolated compounds, particularly compounds $\mathbf{1}, \mathbf{2}, \mathbf{4}$ and $\mathbf{5}$ from active fractions may be responsible for the anti-inflammatory effects of $\mathrm{H}$. microcalycinum as a function of LXRa agonist and free radical scavenging effects.

Also, this is the first study for the isolation of apigenin-8-C-(2-Oacetyl)- $\beta$-glucopyranoside, 3 from a Hypericum species.

Peer-review: Externally peer-reviewed.

Author Contributions: Conception/Design of Study- S.S.A., V.M.K., Ü.Ş.H., I.S.; Data Acquisition- S.S.A., V.M.K.; Data Analysis/Interpretation- S.S.A., V.M.K., T.M., M.I., Ü.Ş.H. I.S.; Drafting Manuscript- S.S.A., V.M.K., Ü.Ş.H.; Critical Revision of Manuscript- V.M.K., T.M., M.I., Ü.Ş.H. I.S.; Final Approval and Accountability- S.S.A., V.M.K., T.M., M.I., Ü.Ş.H. I.S.

Conflict of Interest: The authors have no conflict of interest to declare.

Financial Disclosure: Authors declared no financial support.

\section{REFERENCES}

- $\quad$ Aktas, N., Genc, Y., Gozcelioglu, B., Konuklugil, B., \& Harput, U. S. (2013). Radical scavenging effect of different marine sponges from mediterranean coasts. Records of Natural Products, 7(2), 96104.

- Almahy, H. A., \& Fouda, H. A. R. (2013). Isolation of Luteolin-8-C$\beta$-D-glucopyranoside from the roots of Salvadora persica (Rutaceae). Journal of Current Chemical and Pharmaceutical Sciences, 3, 49-53.

- Baytop, T. (1999). Türkiye'de Bitkilerle Tedavi: Geçmişte ve Bugün, (pp. 166-167). Istanbul, Turkey: İstanbul Üniversitesi Yayınları.

- Boga, M., Ertas, A., Eroglu-Ozkan, E., Kizil, M., Ceken, B., \& Topcu, G. (2016). Phytochemical analysis, antioxidant, antimicrobial, anticholinesterase and DNA protective effects of Hypericum capitatum var. capitatum extracts. South African Journal of Botany, 104, 249-257. doi: 10.1016/j.sajb.2016.02.204

- Donovan, J. L., Luthria, D. L., Stremple, P., \& Waterhouse, A. L. (1999). Analysis of (+)-catechin, (-)-epicatechin and their 3'- and 4 '-O-methylated analogs - A comparison of sensitive methods. Journal of Chromatography B-Analytical Technologies in the Biomedical and Life Sciences, 726(1-2), 277-283. doi: 10.1016/S03784347(99)00019-5

- $\quad$ Eroglu, E., Aksu, B. Z., \& Mat, A. (2008). An overview on Hypericum species of Turkey. Planta Medica, 74(9), 1139-1139.

- Fessler, M. B. (2018). The challenges and promise of targeting the Liver $X$ Receptors for treatment of inflammatory disease. Pharmacology \& Therapeutics, 181, 1-12. doi: 10.1016/j.pharmthera.2017.07.010

- Fobofou, S. A., Franke, K., Sanna, G., Porzel, A., Bullita, E., La Colla, P., \& Wessjohann, L. A. (2015). Isolation and anticancer, anthelminthic, and antiviral (HIV) activity of acylphloroglucinols, and regioselective synthesis of empetrifranzinans from Hypericum roeperianum. Bioorganic \& Medicinal Chemistry, 23(19), 6327-6334. doi: 10.1016/j.bmc.2015.08.028
Fouache, A., Zabaiou, N., De Joussineau, C., Morel, L., SilventePoirot, S., Namsi, A., ... Trousson, A. (2019). Flavonoids differentially modulate liver $X$ receptors activity-Structure-function relationship analysis. The Journal of Steroid Biochemistry and Molecular Biology, 190, 173-182. doi: https://doi.org/10.1016/j.jsbmb.2019.03.028

- Francisco, V., Figueirinha, A., Costa, G., Liberal, J., Ferreira, I., Lopes, M. C., . . Batista, M. T. (2016). The flavone luteolin inhibits liver X receptor activation. Journal of Natural Products, 79(5), 1423-1428. doi: 10.1021/acs.jnatprod.6b00146

- Gong, H., He, J., Lee, J. H., Mallick, E., Gao, X., Li, S., .. Xie, W. (2009). Activation of the liver $X$ receptor prevents lipopolysaccharideinduced lung injury. The Journal of Biological Chemistry, 284(44), 30113-30121. doi: 10.1074/jbc.M109.047753

Hatano, T., Edamatsu, R., Hiramatsu, M., Mori, A., Fujita, Y., Yasuhara, T.,... Okuda, T. (1989). Effects of the interaction of tannins with coexisting substances .6. Effects of tannins and related polyphenols on superoxide anion radical, and on 1,1-diphenyl-2-picrylhydrazyl radical. Chemical \& Pharmaceutical Bulletin, 37(8), 2016-2021.

Hiebl, V., Ladurner, A., Latkolik, S., \& Dirsch, V. M. (2018). Natural products as modulators of the nuclear receptors and metabolic sensors LXR, FXR and RXR. Biotechnology Advances, 36(6), 16571698. doi: https://doi.org/10.1016/j.biotechadv.2018.03.003

- Islam, M., Al-Amin, M. D., Siddiqi, M. M. A., Akter, S., Haque, M. M., Sultana, N., \& Chowdhury, S. (2012). Isolation of quercetin-3-O- $\beta$ D-glucopyranoside from the leaves of Azadirachta indica and antimicrobial and cytotoxic screening of the crude extracts. Dhaka University Journal of Science, 60, 11-14.

- $\quad$ Jensen, S. R., Gotfredsen, C. H., Harput, U. S., \& Saracoglu, I. (2010). Chlorinated iridoid glucosides from Veronica longifolia and their antioxidant activity. Journal of Natural Products, 73(9), 1593-1596. doi: 10.1021/np100366k

- Joseph, S. B., Castrillo, A., Laffitte, B. A., Mangelsdorf, D. J., \& Tontonoz, P. (2003). Reciprocal regulation of inflammation and lipid metabolism by liver $X$ receptors. Nature Medicine, 9(2), 213-219. doi: 10.1038/nm820

- $\quad$ Kadota, S., Takamori, Y., Nyein, K. N., Kikuchi, T., Tanaka, K., \& Ekimoto, H. (1990). Constituents of the leaves of Woodfordia fruticosa Kurz. I. Isolation, structure, and proton and carbon-13 nuclear magnetic resonance signal assignments of woodfruticosin (woodfordin C), an inhibitor of deoxyribonucleic acid topoisomerase II. Chem Pharm Bull (Tokyo), 38(10), 2687-2697. doi: 10.1248/ cpb.38.2687

- Kotani, H., Tanabe, H., Mizukami, H., Makishima, M., \& Inoue, M. (2010). Identification of a naturally occurring rexinoid, honokiol, that activates the retinoid $X$ receptor. Journal of Natural Products, 73(8), 1332-1336. doi: 10.1021/np100120c

Kunchandy, E., \& Rao, M. N. A. (1990). Oxygen radical scavenging activity of curcumin. International Journal of Pharmaceutics, 58(3), 237-240. doi: 10.1016/0378-5173(90)90201-E

- Nakashima, K., Murakami, T., Tanabe, H., \& Inoue, M. (2014). Identification of a naturally occurring retinoid $X$ receptor agonist from Brazilian green propolis. Biochimica et Biophysica Acta-General Subjects, 1840(10), 3034-3041. doi: 10.1016/j.bbagen.2014.06.011 Nicollier, G., \& Thompson, A. C. (1983). Flavonoids of Desmanthus illinoensis. Journal of Natural Products, 46(1), 112-117. doi: 10.1021/np50025a011

Nunez, A. R. (1985). Taxonomia de Hypericum hyssopifolium Chaix Y Especies Relacionadas. Lagascalia, 13(2), 173-185.

- $\quad$ Ohara, K., Wakabayashi, H., Taniguchi, Y., Shindo, K., Yajima, H., \& Yoshida, A. (2013). Quercetin-3-O-glucuronide induces ABCA1 expression by LXRa activation in murine macrophages. Biochemical and Biophysical Research Communications, 441 (4), 929-934. doi: https://doi.org/10.1016/j.bbrc.2013.10.168 
- $\quad$ Prochazkova, D., Bousova, l., \& Wilhelmova, N. (2011). Antioxidant and prooxidant properties of flavonoids. Fitoterapia, 82(4), 513523. doi: 10.1016/j.fitote.2011.01.018

- Razavi, S. M., Zahri, S., Zarrini, G., Nazemiyeh, H., \& Mohammadi, S. (2009). Biological activity of quercetin-3-O-glucoside, a known plant flavonoid. Russian Journal of Bioorganic Chemistry, 35(3), 376-378. doi: 10.1134/S1068162009030133

- Robson, N. K. B. (1980). Hypericum L. . In P. H. Davis (Ed.), Flora of Turkey and East Aegean Islands (pp. 355-401). Edinburgh: Edinburgh University.

- Sarian, M. N., Ahmed, Q. U., So'ad, S. Z. M., Alhassan, A. M., Murugesu, S., Perumal, V., ... Latip, J. (2017). Antioxidant and Antidiabetic Effects of Flavonoids: A Structure-Activity Relationship Based Study. Biomed Research International, 2017. doi: Artn 8386065 doi: 10.1155/2017/8386065

- Srinivasan, R., Chandrasekar, M. J. N., Nanjan, M. J., \& Suresh, B. (2007). Antioxidant activity of Caesalpinia digyna root. Journal of Ethnopharmacology, 113(2), 284-291. doi: 10.1016/j. jep.2007.06.006

- Szondy, Z., Garabuczi, E., Joos, G., Tsay, G. J., \& Sarang, Z. (2014). Impaired clearance of apoptotic cells in chronic inflammatory diseases: therapeutic implications. Frontiers in Immunology, 5, 354. doi: 10.3389/fimmu.2014.00354

- Tsai, P. J., Tsai, T. H., Yu, C. H., \& Ho, S. C. (2007). Comparison of NOscavenging and NO-suppressing activities of different herbal teas with those of green tea. Food Chemistry, 103(1), 181-187. doi: 10.1016/j.foodchem.2006.08.013

- Vedin, L. L., Gustafsson, J. A., \& Steffensen, K. R. (2013). The oxysterol receptors LXRalpha and LXRbeta suppress proliferation in the colon. Molecular Carcinogenesis, 52(11), 835-844. doi: 10.1002/ mc.21924
Viennois, E., Mouzat, K., Dufour, J., Morel, L., Lobaccaro, J. M., \& Baron, S. (2012). Selective liver X receptor modulators (SLiMs): What use in human health? Molecular and Cellular Endocrinology, 351(2), 129-141. doi: 10.1016/j.mce.2011.08.036

- Wang, W., Nakashima, K., Hirai, T., \& Inoue, M. (2019). Anti-inflammatory effects of naturally occurring retinoid $X$ receptor agonists isolated from Sophora tonkinensis Gagnep. via retinoid X receptor/liver $X$ receptor heterodimers. Journal of Natural Medicines, 73(2), 419-430. doi: 10.1007/s11418-018-01277-1

- Willy, P. J., Umesono, K., Ong, E. S., Evans, R. M., Heyman, R. A., \& Mangelsdorf, D. J. (1995). Lxr, a Nuclear Receptor That Defines a Distinct Retinoid Response Pathway. Genes \& Development, 9(9), 1033-1045. doi: 10.1101/gad.9.9.1033

- Zhang, P. C., \& Xu, S. X. (2003). C-glucoside flavonoids from the leaves of Crataegus pinnatifida Bge. var. major N.E.Br. Journal of Asian Natural Products Research, 5(2), 131-136. doi: 10.1080/1028602021000054982

- Zhang, X. F., Thuong, P. T., Jin, W., Su, N. D., Sok, D. E., Bae, K., \& Kang, S. S. (2005). Antioxidant activity of anthraquinones and flavonoids from flower of Reynoutria sachalinensis. Archives of Pharmacal Research, 28(1), 22-27. doi: 10.1007/Bf02975130

- Zorzetto, C., Sanchez-Mateo, C. C., Rabanal, R. M., Lupidi, G., Petrelli, D., Vitali, L. A., ... Maggi, F. (2015). Phytochemical analysis and in vitro biological activity of three Hypericum species from the Canary Islands (Hypericum reflexum, Hypericum canariense and Hypericum grandifolium). Fitoterapia, 100, 95-109. 